\title{
Ambiente y extensión en Paso Centurión, Uruguay: la construcción de una práctica integral y los caminos hacia el diálogo de saberes
}

\section{Carlos Santos}

Centro Universitario Regional del Este, Facultad de Ciencias Sociales.

carlos.santos@cienciassociales.edu.uy

(iD) orcid.org/0000-0002-1632-8129

\author{
Magdalena Chouhy \\ Facultad de Humanidades y Ciencias \\ de la Educación. \\ magdalenachouhy@gmail.com \\ (iD) orcid.org/0000-0001-7308-1145
}

RECEPCIÓN: 30/04/21

ACEPTACIÓN FINAL: 27/05/21

\section{Resumen}

Este artículo recoge reflexiones de un equipo que realiza extensión universitaria desde una perspectiva integral sobre las relaciones sociedad-naturaleza en un área protegida del noreste de Uruguay. El equipo integrado por personas con formación de base en biología y antropología desarrolla desde 2013 una propuesta de formación de grado para diferentes carreras de la Universidad de la República como plataforma a partir de la cual se han desplegado diferentes acciones de vínculo con el medio y se ha abierto camino para procesos de diálogo de saberes con pobladores locales. De manera transversal, desde el inicio de la experiencia ha habido un componente permanente y sostenido de producción de conocimiento que se ha vehiculizado en diferentes estrategias (investigaciones estudiantiles en el marco del curso, presentaciones del equipo docente en otros cursos y eventos científicos, artículos, tesis de grado y de posgrado, proyectos de investigación) que permiten hoy reconstruir una trayectoria integral.

Palabras clave: integralidad; relaciones sociedad-naturaleza; diálogo de saberes; Paso Centurión; Uruguay.
Lucía Bergós

Centro Universitario Regional del Este. lucia.bergos@gmail.com

iD orcid.org/0000-0003-2330-3808

Universidad de la República, Uruguay

Andrea Garay

Universidad Tecnológica, Uruguay.

andrealgaray@gmail.com

(iD) orcid.org/0000-0001-7547-9405

Environment and extensionism in Paso Centurión, Uruguay: building an integral practice and paths towards the dialogue of knowledge

\section{Abstract}

This article collects reflections of a university team that carries out extensionism from an integral perspective on society-nature relations in a protected area in the northeast of Uruguay. Since 2013 the team integrated, mainly, by biologists and anthropologists has organized an interdisciplinary undergraduate training seminar at Universidad de la República, thought as a platform from which different actions have been deployed to outreach local people while cultivating processes of dialogue of knowledge. From the beginning of the experience there has been a permanent and sustained effort of scientific knowledge production that is conveyed in different strategies, namely undergraduate students research proposals, lecturers' participation in other training courses and academic events, submission of articles, elaboration of graduate and postgraduate thesis and R\&D projects. All this production allows today to acknowledge an integral trajectory.

Keywords: integrality; society-nature relations; dialogue of knowledges; Paso Centurión; Uruguay.
Ambiente y extensión universitaria /

Desafíos de gestión

다(1)(2)

\author{
Meio ambiente e extensão em Paso \\ Centurión, Uruguay: a construção de uma \\ prática integral e os caminhos para o \\ diálogo de saberes
}

\section{Resumo}

Este artigo reúne reflexões de uma equipe que realiza extensão universitária sob uma perspectiva integral das relações entre a sociedade e a natureza em uma área protegida no nordeste do Uruguai. Desde 2013, a equipe formada por pessoas com formação básica em biologia e antropologia desenvolveu uma proposta de formação de grau para diferentes cursos na Universidade de la República como uma plataforma a partir da qual têm se desdobrado diferentes ações de vinculação com o meio ambiente e de apertura para processos de diálogo de saberes com a população local. De forma transversal, desde o início da experiência tem havido um componente permanente de produção de conhecimento que tem se veiculado com diferentes estratégias (pesquisas dos alunos no âmbito da aula, apresentações da equipe docente em outras aulas e eventos científicos, artigos, teses de graduação e pós-graduação, projetos de pesquisa) que permitem hoje reconstruir uma formação integral.

Palavras-chave: integralidade; relações sociedade-natureza; diálogo de saberes; Paso Centurión; Uruguai.

Para citación de este artículo: Santos, C.; Chouhy, M.; Bergós, L. y Garay, A. (2021). Ambiente y extensión en Paso Centurión, Uruguay: la construcción de una práctica integral y los caminos hacia el diálogo de saberes. +E: Revista de Extensión Universitaria, 11(14), e0002. doi: 10.14409/extension.2021.14.Ene-Jun.e0002 


\section{Introducción ${ }^{1}$}

Existen múltiples aproximaciones para discutir el cruce entre los procesos de extensión universitaria y las problemáticas o cuestiones ambientales. En el caso que se expone, de la Universidad de la República de Uruguay (UdelaR), el contexto para el desarrollo de la extensión ha estado marcado en la última década por la noción de integralidad (Tommasino, Cano, Castro, Santos y Stevenazzi, 2010), esto es: 1) la articulación de las funciones de investigación, enseñanza y extensión; 2) la interdisciplinariedad; y 3) el diálogo entre saberes académicos y saberes populares. El eje articulador del trabajo de este equipo es un espacio curricular llamado Espacio de Formación Integral (EFI) "Relaciones sociedad-naturaleza en la frontera", que se desarrolla desde 2013 en Paso Centurión, una localidad rural en el departamento de Cerro Largo, en la región noreste de Uruguay, casi sobre la línea de frontera con el Brasil, frontera político-administrativa establecida por el río Yaguarón. El EFI es una propuesta de formación de grado abierta para estudiantes de diferentes carreras de la UdelaR como plataforma a partir de la cual se han desplegado diferentes acciones de vínculo con el medio y se ha abierto camino para procesos de diálogo de saberes con pobladores locales.

Este artículo desarrolla la manera en que se ha llevado adelante el proceso de construcción de la demanda (Barrero, Cardozo, González, Grabino, Viñar, y Santos, 2015; Retamozo, 2015; Merlinsky, 2018) entre el equipo universitario y los actores locales de Paso Centurión. En particular, esta construcción de la demanda ha estado centrada en los efectos de la ambientalización ${ }^{2}$ del territorio de Paso Centurión. El área es objeto de una serie de intervenciones de instituciones y organizaciones ambientalistas así como de una política de conservación de la naturaleza (el establecimiento de un área protegida), por lo cual se ha desarrollado un conflicto ambiental. ${ }^{3}$ Compartimos, como señala Merlinsky (2018), que durante procesos de extensión integrales en contextos de conflictos ambientales no basta con aportar el conocimiento experto ni aun la generación de nuevos conocimientos de esta clase, sino que

"el saldo más interesante es que se generan nuevas experticias sobre el territorio. Los pobladores hacen valer su conocimiento y se autoperciben con autoridad para reclamar y oponerse a las amenazas (...) De este modo se asumen como portadores de un conocimiento válido que abre interrogantes sobre la calidad de vida, la salud y el bienestar de la comunidad". (p. 259)

1) Son colaboradores de este trabajo en pie de igualdad Javier Taks y Gabriel Perazza, integrantes del equipo docente. 2) La noción de ambientalización de la sociedad o de los conflictos sociales ha surgido en la emergencia de lo que algunos llaman una nueva cuestión social: muchas veces, sostienen algunos autores, los conflictos ambientales son conflictos sociales cuyos motivos subyacen a la temática ambiental que los hace evidentes (Leite Lopes, 2006; Taks, 2013; Ferrero, 2013). Este carácter ambientalizado está dado por la construcción de una nueva cuestión social, una nueva cuestión política que tiene su foco en lo ambiental (Leite Lopes, 2006).

3) Entre 2011 y 2013, pobladores de Paso Centurión desplegaron una serie de acciones de rechazo a la implantación de monocultivos de árboles en la zona con destino a la producción de pulpa de celulosa. La movilización generó algunas intervenciones del gobierno departamental con moratorias a los cultivos que culminaron con la categorización de toda la zona como suelo rural natural en el instrumento de Ordenamiento Territorial aprobado por la Junta Departamental en 2016. Más recientemente se produjo un nuevo foco de conflictividad ambiental a partir de su ingreso al Sistema Nacional de Áreas Protegidas, ya que un número importante de empresarios agropecuarios dueños de amplias extensiones de tierra inciaron acciones jurídicas para revertir estas regulaciones y poder disponer libremente del uso de sus establecimientos (Santos, 2020). 
Paso Centurión y su vecina Sierra de Ríos forman parte de un área considerada de interés para la conservación por su rica biodiversidad, declarada Reserva Departamental en 2007 e ingresada al Sistema Nacional de Áreas Protegidas en 2019. ${ }^{4}$ Entre otros aspectos destacados de su biodiversidad, se reconoce como una zona con alta concentración de registros de mamíferos prioritarios para la conservación en Uruguay (Grattarola y RodríguezTricot, 2020). Por otra parte, está históricamente dedicada a la ganadería extensiva, que presenta niveles socioeconómicos críticos en términos de pobreza en zonas rurales ${ }^{5}$ y atraviesa un proceso de despoblamiento, el que la población local atribuye a múltiples causas: falta de ofertas en educación media, escasez de fuentes de empleo y emigración de familias productoras como consecuencia de los cambios en el uso del suelo, ya que se pasó de la actividad agropecuaria tradicional a la forestal industrial. ${ }^{6}$

Ciertas características de la configuración social de la zona plantean algunas dificultades para el trabajo universitario. Por un lado, en los primeros años de la intervención no existían colectivos sociales consolidados que pudieran sostener una carga de trabajo local como contraparte. Por otro lado, en su mayoría se trata de población rural envejecida y dispersa, de la cual un gran número de varones reside en sus lugares de trabajo, alejados de los núcleos poblados durante la mayor parte del tiempo. La manera de planificar, pensar y sostener la participación social tuvo que adaptarse a estas condiciones y tratar de crear las circunstancias requeridas para desarrollar un proceso participativo (Fals Borda, 1981).

En ese sentido, el trabajo realizado siempre con la intención de revalorizar el saber local frente a la declinación de la "arrogancia del experto" (en términos de Fals Borda) fue una de las estrategias que permitió pensar las actividades en colaboración entre el equipo universitario y la población local.

El EFI "Relaciones Sociedad-Naturaleza en la frontera" se originó a partir del vínculo establecido entre la Asociación Civil JULANA (Jugando en la Naturaleza), un colectivo principalmente conformado por profesionales de las ciencias naturales que desarrolla acciones de Educación Ambiental en la localidad de Paso Centurión. Desde integrantes de la organización con vínculo con la UdelaR surgió la necesidad de consolidar un espacio formativo en el cual el trabajo con la comunidad local enriqueciera la formación de los estudiantes universitarios y, a la vez, favoreciera a la población local a partir del intercambio y del conocimiento generado en ese marco (Santos et al., 2020).

Desde su primera edición, en el año 2013, el EFI ha incluido los tres niveles constitutivos de la integralidad. Articula las funciones universitarias, ya que es un espacio formativo (enseñanza) en el que se produce conocimiento (investigación), en colaboración entre actores universitarios y no universitarios (extensión); integra a su vez docentes y estudiantes de

4) Con respecto al Área Protegida, se comienza a desarrollar otro conflicto debido al interés de recortar su extensión por parte de sectores privados (La voz de Melo, 2021).

5) Integró la zona de intervención del Prodenor (Proyecto de Desarrollo Social de las Zonas Rurales más Pobres del Norte de Uruguay), que trabajó en 18 zonas de Cerro Largo y en el área este de Salto y de Artigas, entre 2005 y 2008 , a través del Ministerio de Vivienda, Ordenamiento Territorial y Medio Ambiente (MVOTMA) y de la Comisión Honoraria Pro Erradicación de la Vivienda Rural Insalubre (MEVIR).

6) El $78 \%$ de los suelos es de prioridad forestal y en los últimos años han aumentado las inversiones para la forestación. No obstante, en 2010 el avance de la actividad forestal se vio detenido por medidas cautelares de la Junta Departamental en virtud del carácter de Reserva Departamental. Esto respondió a la iniciativa de pobladores locales, quienes entendían que la forestación era incompatible con la práctica ganadera y el modo de vida tradicional. 
diferentes procedencias disciplinares (interdisciplinariedad), y promueve que los procesos formativos, de investigación y de extensión ocurran en un marco de intercambio de conocimientos - académicos y locales-que explicite las diferentes miradas y fomente la reflexión constante sobre ese vínculo.

En su abordaje, este equipo no partió de una definición disciplinar de ambiente sino que en todo caso se trató de seguir la sugerencia de Ingold de:

"pensar al ambiente como una zona de interpenetración [entre humanos y no humanos] (...) continuamente en obra, continuamente creciendo al tiempo que los habitantes del ambiente hacen sus caminos a través, siguiendo diferentes sendas". (2012, p. 73)

Este punto de partida habilitó el lugar de las diferentes disciplinas y más tarde los diversos saberes en torno a esa construcción permanente del ambiente. En el trabajo de campo del equipo esta apertura conceptual con relación a la noción de ambiente permitió intervenir sin partir desde una concepción unívoca de ambiente asociada a la reciente ambientalización del área y las intervenciones desde la conservación.

Hacer preguntas recíprocas sobre los conceptos, exponer los saberes propios como conocimientos por completar, hablar de las propias "ignorancias" para habilitar el conocimiento del otro, fueron elementos fundamentales para transitar hacia la interdisciplina y posibilitar el diálogo de saberes en el campo. La ignorancia es aquí entendida no como un elemento negativo sino como característica intrínseca a cualquier proceso de producción de conocimiento (Feyerabend, 1998; Pombo, 2013). En un sentido similar pero enfocado a su propuesta de una ecología de saberes, Boaventura de Sousa Santos sostiene que un paso previo para el reconocimiento de los saberes de las otras personas es el diálogo de sus ignorancias:

"En la ecología de los saberes, los conocimientos interactúan, se entrecruzan y, por tanto, también lo hacen las ignorancias. Tal y como allí no hay unidad de conocimientos, tampoco hay unidad de ignorancia. Las formas de ignorancia son tan heterogéneas e interdependientes como las formas de conocimiento. Dada esta interdependencia, el aprender determinadas formas de conocimiento puede implicar olvidar otras $\mathrm{y}$, en última instancia, convertirse en ignorantes de las mismas. En otras palabras, en la ecología de saberes la ignorancia no es necesariamente el estado original o el punto de partida. Este podría ser un punto de llegada. Podría ser el resultado del olvido o del olvidar implícito en el proceso de aprendizaje recíproco". (Santos, 2009, p. 185)

\section{Prácticas en movimiento}

Desde sus inicios, el equipo universitario se planteó una colaboración horizontal y permanente con la organización JULANA. Asimismo, ha estado vinculado con diferentes pobladores locales y algunos de sus colectivos conformados en los últimos años: un grupo de aspirantes a tierras del Instituto Nacional de Colonización, emprendimientos ecoturísticos y, en particular, un colectivo de mujeres denominado Las Quitanderas, que desarrolla servicios de guía de turismo, alojamiento y alimentación para visitantes. En términos institucionales, la mayor aproximación de colaboración se ha establecido con la guardaparques del área, si bien también existen diferentes niveles de comunicación con el personal de los gobiernos municipal, departamental y nacional con incidencia en Paso Centurión. 
En los ocho años transcurridos, el equipo desarrolló diferentes acciones de vinculación con la población local en el marco de las iniciativas de extensión universitaria. En un primer momento, el EFI se complementó con la iniciativa del monitoreo participativo de fauna con cámaras trampa ${ }^{7}$ llevada adelante en Paso Centurión por JULANA junto a pobladores y pobladoras de la zona. En ese entonces se realizó una colecta de fecas con posterior análisis de ADN con el objetivo de identificar presencia de mamíferos relevantes para la conservación a través de un muestreo sistemático (Grattarola et al., 2014; Chouhy et al., 2017). En paralelo con este proceso y como parte de un abordaje social, se trabajó en la revalorización de los saberes locales asociados a la fauna, incluyendo conocimiento de las especies, hábitos de alimentación o de refugio, anécdotas, intentos de domesticación y recetas de preparación de alimentos. Posteriormente, el equipo trabajó en la producción de conocimientos que permitieran generar evidencias no solo sobre la presencia de especies prioritarias para la conservación sino también sobre procesos sociales y económicos de la zona. En ese sentido, se generó evidencia sobre cambios en el uso del suelo y nuevas actividades económicas, por ejemplo, forestación industrial de eucalipto, producción industrial de energía eólica y un incipiente ecoturismo (Bergós et al., 2019).

Acompañando todo el período de intervención se llevaron a cabo procesos de formación de estudiantes de grado y de posgrado que incluyeron tesis en ambos niveles en las cuales se abordaron temáticas referidas a relaciones humanos-animales, conflictos ambientales e implementación de políticas de conservación de la naturaleza. Una de las acciones permanentes del equipo tiene que ver con la comunicación de resultados y el mantenimiento "al día" de las acciones desplegadas con aquellos actores locales con los que existe un mayor vínculo. Esas estrategias contemplan:

a) Compartir el registro de las cámaras trampa del monitoreo participativo con las personas directamente participantes de la tarea.

b) Ampliar la audiencia de los registros de las cámaras trampa a través de proyecciones públicas, por ejemplo, en la Escuela № 16 de Paso Centurión.

c) Presentar pósteres o resúmenes del trabajo de cada generación de estudiantes del EFI en coincidencia con la realización de la fiesta de fin de curso de la mencionada escuela.

d) Realizar actividades lúdicas de intercambio y comunicación de hitos en el proceso de producción de conocimiento.

e) Llevar a cabo talleres para acercar información específica a partir de la demanda de actores locales.

f) Entablar comunicación permanente con personas referentes de la población de Paso Centurión a través de JULANA.

g) Realizar y luego proyectar un audiovisual que documenta el trabajo del equipo universitario y JULANA en la zona.

Todas estas estrategias operan respecto de mantener a la población local en conocimiento de las acciones desplegadas, sus resultados y la discusión colectiva de la orientación

7) Las cámaras trampa son dispositivos remotos que integran una cámara fotográfica digital con sensores de movimiento y calor que permiten fotografiar o filmar en video a animales cuando pasan por delante de la cámara (Bergós et al., 2018). 
general de esas acciones. La idea de devolución sistemática la tomamos de Fals Borda en tanto que herramienta de "restitución sistemática" que tiene la finalidad de "facilitar la apropiación social del conocimiento" (2014, p. 245).

\section{Mojones en la construcción de un diálogo de saberes}

Como hilo conector del proceso general de esta trayectoria integral y en el contexto de este proceso de devolución sistemática que se mencionaba, el equipo desarrolló una serie de acciones que podríamos hoy día llamar "mojones de la trayectoria", las cuales oportunamente fueron apoyadas en el marco de la Convocatoria a Actividades en el Medio de la Comisión Sectorial de Extensión y Actividades en el Medio de la UdelaR. Se trata de una convocatoria que apoya acciones puntuales en el tiempo y el espacio con un monto limitado para cubrir gastos operativos. $^{8}$

\section{Devolución del monitoreo con cámaras trampa}

En el segundo año de la intervención en Paso Centurión, en 2014, se llevó a cabo una actividad que consistió explícitamente en la devolución y discusión de los resultados del trabajo del EFI en el año anterior. ${ }^{9}$ Esta actividad involucró tanto al equipo universitario como a la organización JULANA y a la población local. En cuanto al desarrollo del monitoreo participativo de fauna de la zona, se centró en el trabajo con las comunidades educativas, las escuelas de la zona ( $\mathrm{N}^{\circ} 16$ y $\mathrm{N}^{\circ}$ 25). Y en la instancia de devolución se logró integrar las técnicas de muestreo de fecas y el abordaje social a partir de entrevistas abiertas y en profundidad. Como resultado se pudo sostener el vínculo con pobladores locales y docentes responsables de las escuelas así como identificar intereses y posibilidades de continuidad de la propuesta a futuro. Surgieron ideas a realizar en el marco del EFI, como talleres o encuentros donde compartir informalmente saberes (historias y leyendas) sobre la fauna local. El evento en sí logró expandir los resultados de la presencia universitaria hacia las personas que no habían participado directamente de las actividades desplegadas.

\section{Taller historias de fauna}

En 2015 se desarrolló otra actividad enfocada en los acuerdos alcanzados el año anterior. En esta ocasión se trató de un taller sobre las historias y leyendas locales con relación a la fauna. Se realizó en la Escuela №16 pero se convocó a vecinos y vecinas de la zona de todas las generaciones. La propuesta se articuló con el proyecto "Fogones de Fauna" de JULANA (Bergós et al., 2018) y buscó rescatar la importancia de la transmisión oral del saber popular e identificarlo como otra forma de registro, al igual que los registros de fauna con cámaras trampa. Esta articulación fue una oportunidad para conocer el alcance del trabajo desplegado en la zona por JULANA y acercarse a la población local en un ambiente de confianza.

8) "En un sentido amplio, las actividades en el medio comprenden las acciones llevadas adelante por universitarios en vínculo con población no universitaria, a través de actividades de asistencia, difusión o divulgación científica y procesos de transferencia tecnológica. La modalidad también presenta un instrumento para el desarrollo de actividades puntuales, enmarcadas en procesos de extensión" (UdelaR, 2019, p. 1).

9) "Devolución y reflexión colectiva sobre resultados obtenidos en el marco del EFI "Monitoreo Participativo de Fauna en Paso Centurión, Cerro Largo". 
Durante la actividad, grupos integrados por vecinos y vecinas de Paso Centurión, miembros de JULANA y docentes y estudiantes del EFI trabajaron sobre la base de la siguiente consigna: las personas integrantes del grupo debían narrar una historia ocurrida en el lugar vinculada a un animal o entidad del imaginario colectivo (entidades de carácter mágico denominadas "asombros" que surgieron espontáneamente a partir de la interpretación de la consigna por parte de los habitantes). Luego se seleccionaba una historia para representar ante el resto de los grupos. De esta manera se pudieron recopilar historias de forma escrita (ya que cada equipo debía escribir la suya a modo de guion), así como compartir múltiples percepciones sobre las escenificaciones presenciadas. La propuesta fue evaluada como altamente positiva por todas las personas involucradas. Surgieron de esta actividad numerosas iniciativas para dar continuidad al trabajo en la zona, muchas de ellas propuestas por los estudiantes del EFI. El involucramiento de las y los estudiantes con la propuesta y con la comunidad fue un aspecto especialmente destacado por docentes y estudiantes del EFI.

\section{Video-foro junto al río Yaguarón}

Al año siguiente (2016), el equipo universitario realizó otra actividad en la que se propuso la dinamización de un cine-foro en torno a la presentación de un documental sobre el monitoreo participativo de JULANA en Paso Centurión. ${ }^{10}$ Se superaron ampliamente las expectativas en cuanto a la concurrencia: participaron más de 200 personas, y la mayoría de ellas se trasladaron desde la ciudad de Melo (capital departamental ubicada a $60 \mathrm{~km}$ de Paso Centurión) en vehículos colectivos contratados para la ocasión. Esto, sumado a que la actividad se hacía al aire libre (en las ruinas de una vieja aduana a orillas del río Yaguarón), obligó a una reformulación de la propuesta inicial, ya que se consideró inviable la modalidad de taller o foro. La estrategia alternativa desarrollada fue dividir al equipo universitario, miembros de JULANA y documentalistas en pequeños equipos que entrevistaron brevemente a los espectadores para conocer sus opiniones e intercambiar sobre el documental presentado.

\section{Encuentro de saberes}

En 2017, previo al inicio de una nueva edición del EFI se realizó una actividad denominada "Encuentro de saberes en Paso Centurión". Fue el primer paso que se dio en la dimensión que el equipo entendía como la menos abordada en forma explícita en el intercambio con la población de Paso Centurión, de aquellas constitutivas de la integralidad: el diálogo de saberes. La actividad consistió en un seminario de intercambio de saberes académicos y saberes populares sobre qué es el conocimiento situado. Allí el equipo universitario se explayó acerca de los trayectos que había tomado el conocimiento construido con base en la intervención e investigación en Paso Centurión - a través de informes de estudiantes, ponencias en congresos en Uruguay y en la región, artículos de prensa y en revistas académicas, tesis de grado y posgrado-y la importancia de dicho trabajo en términos de formación para los universitarios participantes del proceso. De esta manera se transparentó nuestro papel como actores del campo académico (Fernández Álvarez y Carenzo, 2012) y se dio también una idea del volumen de la producción resultante de nuestro trabajo en el lugar y de los ámbitos académicos donde esta se comparte.

10) Documental Jugando en la Naturaleza. Disponible en: https://archive.org/details/jugando_en_la_naturaleza bajo licencia Creative Commons. 
Como paso previo, el equipo organizó un encuentro en Montevideo en el cual el equipo docente, exestudiantes de todas las ediciones del EFI, miembros de JULANA y tesistas de posgrado con locus en Centurión compartieron trayectorias, proyectos y ejes de trabajo en el área. Esto fue importante para dimensionar y ordenar aquello que se compartiría más adelante en la localidad sobre el propio trabajo académico. En la jornada también se hicieron propuestas de ejes temáticos globalizadores para llevar adelante el seminario en Paso Centurión, así como propuestas didácticas.

Durante el taller en Paso Centurión que se denominó "Encuentro de Saberes", a partir de lo mencionado sobre las producciones académicas, se impulsó un intercambio sobre las diferentes visiones del proceso de intervención que comenzó en el año 2013, sus repercusiones en las personas involucradas y en el área de trabajo. Se formaron grupos para discutir en torno a los conceptos de información, conocimiento, y a cómo los diferentes saberes se articulan en el ámbito académico y local.

Luego cada grupo preparó una representación teatral que presentó una situación en la que se daba un diálogo entre saberes locales y académicos respecto de temas ambientales. Estas piezas teatrales semiimprovisadas fueron guionadas por los habitantes de Paso Centurión al interior de cada grupo. De forma lúdica y teatral, se trabajó sobre el conocimiento ambiental local y científico y sobre cómo estos pueden interactuar y colaborar entre sí. La idea de una integración de saberes fue puesta en debate. La atención del equipo universitario se centró especialmente en cómo los tipos de conocimientos y sus interacciones eran categorizados y representados por los pobladores participantes.

Esta actividad permitió, por un lado, explicitar la intencionalidad del equipo universitario de establecer una dinámica de trabajo integral sobre ambiente y territorio en Paso Centurión, buscando para eso articular un diálogo de saberes entre pobladores, colectivos, instituciones y equipo universitario. Por otro lado, mediante las dinámicas utilizadas, las personas de Paso Centurión compartieron sus ideas sobre la producción de conocimientos científicos (en particular de la biología de campo), sobre el conocimiento local y posibles interrelaciones entre estos. Estas ideas, objetivadas y escenificadas en un clima de confianza, fueron esenciales para saber cómo en ese momento eran percibidas las prácticas científicas "clásicas" y conocer la valoración de sus propios conocimientos.

\section{Lenguajes de valoración y conflictos ambientales en la extensión universitaria}

Esta trayectoria atraviesa distintas experiencias y reflexiones que van reformulando el diálogo de saberes, las metodologías y los lenguajes utilizados en la práctica de integralidad. A través de un recorrido por las actividades en el medio realizadas entre 2014 y 2017 en Paso Centurión es posible reconocer y objetivar aspectos de los distintos momentos en este proceso de construcción. Las diferentes experiencias dieron lugar a reflexiones que fueron claves para el desarrollo de las siguientes actividades planificadas por el equipo en el territorio, incluyendo las reformulaciones del EFI luego de cada edición. El equipo logró, en cada actividad realizada, por una parte, consolidar una narrativa de los pasos ya realizados $\mathrm{y}$, por otra, mejorar los dispositivos para avanzar hacia un más profundo intercambio de saberes académicos y no académicos. 
Se destaca la herramienta del taller, cuyo potencial para el intercambio de saberes y la promoción de la reflexión crítica (Fernández Álvarez y Carenzo, 2012; Ghiso, 1999) se irá aprovechando en manera creciente. ${ }^{11}$ Con el sostenimiento de la intervención a lo largo del tiempo y la construcción de vínculos de confianza (lo que incluye y depende de la articulación con JULANA), se va transformando la cantidad y la calidad de la participación de las personas de Paso Centurión. Es notable que las personas que al inicio no tomaban fácilmente la palabra en este tipo de instancias lleguen a expresarse de modo fluido e incluso a "desplegar una actuación" frente a un grupo heterogéneo. En consonancia, destaca asimismo la potencialidad del taller como fuente de datos que, al combinarse con la observación participante y las entrevistas, va formando un importante material sobre múltiples aspectos del lugar y sus habitantes.

La actividad de 2014, que consistió en una devolución de resultados del EFI 2013, permitió poner en práctica, en términos ya citados de Fals Borda (2014, p. 245), una "herramienta de restitución sistemática" que facilita "la apropiación social del conocimiento".

El Encuentro de Saberes de 2017, donde junto a la población local se escenificó y debatió sobre las prácticas científicas y los conocimientos locales, significó un paso más hacia el diálogo de saberes. Pudo verse en acción lo señalado más arriba: el potencial de la herramienta del taller para el intercambio de saberes y la reflexión crítica. De todas maneras, en retrospectiva, parece claro que tanto la idea como lo que aconteció en el propio taller fueron posibles por las condiciones ya mencionadas dadas por la temporalidad larga de la intervención: la confianza generada, la acumulación de experiencias en el terreno, la formación teórica y la actitud reflexiva del equipo sobre su práctica, en clave de integralidad.

A partir de la práctica en el tiempo se identificaron también las limitaciones del taller como herramienta metodológica. Desde el comienzo se evidenció la necesidad de combinar estas importantes instancias colectivas con otras más reducidas, como las visitas a familias o personas y las reuniones menos numerosas pero de gran intensidad, menos estructuradas y flexibles a los tiempos y rutinas de los y las habitantes. Estas alternativas metodológicas, que en principio se plantearon a raíz de la inexistencia de colectivos sociales consolidados durante esos años de trabajo y de que muchas personas prefieren no participar en reuniones o talleres, se vuelven clave de la intervención social.

Por otro lado, la visualización en conjunto de registros de cámaras trampa como forma de aprendizaje colaborativo, que es el fundamento del monitoreo participativo desarrollado por JULANA, se muestra desde el inicio como una potente herramienta para la práctica integral del equipo universitario. Este monitoreo será eje vertebral de la trayectoria en torno a un doble vínculo: con la organización civil y con la población local. La visualización de fotos y videos de animales en las casas donde se encuentran las cámaras y en reuniones en la escuela ha permitido la dinamización de los intercambios así como la socialización de los innumerables registros de fauna, entre los que se destaca uno de los registros más recientes de aguará guazú para y el primer registro de un yaguarundí para Uruguay (Grattarola et al.,

11) Ghiso reconoce que hay un menor desarrollo del taller como dispositivo para la producción de conocimientos, y que es mayormente valorado como "un instrumento válido para la socialización, la transferencia, la apropiación y el desarrollo de conocimientos, actitudes y competencias de una manera participativa y pertinente a las necesidades y cultura de los participantes" (1999, p. 142). 
2016; Grattarola y Rodríguez-Tricot, 2020). Esta forma de producción y transmisión de conocimientos ambientales se contrapone a aquellas prácticas clásicas de investigación científica en la que la población local participa a lo sumo en calidad de guía en el trabajo de campo, prestadora de servicios e incluso informante que son luego invisibilizados en las producciones académicas.

Los intercambios en el marco del monitoreo ponen en juego saberes científicos y no científicos y se genera así un espacio fértil para el diálogo de conocimientos, objetivo que el equipo universitario y JULANA irán poniendo de relieve con creciente intensidad. Allí también convergen distintas sensorialidades, porque más allá de lo visual que proponen "las pantallas" (denominación que asignan las personas de Paso Centurión a las cámaras trampa), la oralidad es central. Los relatos compartidos parten de experiencias entre personas y animales que involucran todos los sentidos, narrativas que van configurando distintos conocimientos. La actividad de 2016, en la que se representaron historias sobre animales, fue un hito importante en el reconocimiento de los saberes locales en un mismo nivel que los científicos. Asimismo, la oralidad y el juego se identificaron como formas de registro y transmisión de conocimientos.

En tal sentido, el monitoreo se conforma como medio para el diálogo de saberes (entre la población de Paso Centurión, integrantes de JULANA y el equipo universitario), ya que permite el intercambio de historias, experiencias y conocimientos. Este intercambio no solo acontece en torno a la fauna nativa y silvestre sino también a los animales domésticos y productivos, relevantes en el modo de vida ligado la producción ganadera en el lugar.

La búsqueda del diálogo de saberes sobre temas socioambientales sin una definición restringida de lo ambiental, sino intentando comprender cómo los habitantes perciben lo que "acordamos llamar ambiente" (Ingold, 2012, p. 65) hizo factible la emergencia de otros temas. Nuevos problemas de investigación e intervención comenzaron a ser trabajados por estudiantes en el EFI, proyectos estudiantiles de investigación y extensión (Quintana, Capurro y González Fajardo, 2019), tesis de posgrado y proyectos de investigación desarrollados por este equipo (Bergós et al., 2019). De este modo, los temas de investigación e intervención fueron crecientemente conectados a las inquietudes, demandas y problemáticas definidas a partir del diálogo de saberes. Estas distintas prácticas universitarias profundizan en dicho diálogo a través de innovaciones educativas y metodológicas en el marco de la integralidad y con el objetivo de coproducir conocimientos junto a otros.

La coproducción de conocimientos busca incorporar a los actores sociales como protagonistas activos en la investigación de los procesos que los involucran, y puede darse no solo en el marco de investigaciones participativas sino a partir de diálogos de saberes, procesos interdisciplinarios y extensión universitaria (Santos, Prol y Bergós, 2019). En el caso que se presenta en este artículo, las actividades de investigación, enseñanza y extensión, así como el diálogo de saberes se producen en el contexto de situaciones de conflictividad ambiental (dada por el avance de monocultivos forestales e instalación de parques eólicos) (Santos, Prol y Bergós, 2019), aunque estos no son conceptualizados por los actores locales en términos ambientales.

A pesar de que desde sus inicios esta intervención ha estado fundamentada en una problemática ambiental (las relaciones sociedad-naturaleza) y eso ha sido explicitado con los interlocutores, estos nunca se han autodefinido como ambientalistas o personas con preocupaciones por el ambiente. Estas categorías son propias de la visión académica so- 
bre las relaciones sociedad-naturaleza y cobran otros sentidos al ser consideradas por los pobladores de Paso Centurión.

Como sostiene Merlinsky, "los mismos actores son reacios a considerarse a sí mismos como 'ecologistas'” (2018, p. 242), lo que según la autora remite en realidad a la existencia de diferentes lenguajes de valoración ${ }^{12}$ (Martínez-Alier, 2006). Una de las primeras evidencias de esos lenguajes de contradicción diversos y antagónicos fue la constatación del rechazo de los pobladores locales a iniciativas de plantación de eucaliptos en la zona bajo la encendida defensa de una forma de vida local (Santos, C. 2020). En ese sentido, emergió ante la mirada y la escucha del equipo la manera en que los pobladores locales denominaban a los monocultivos de árboles: "florestación". Propio de la diglosia lingüística de la frontera, donde los dialectos portugueses del Uruguay conviven con el castellano y el portugués, este proceso de avance de los (mono)cultivos forestales, la silvicultura, como deberíamos llamarle (Gautreau, 2014), es nominada por los pobladores con ese término. A mitad de camino entre la "floresta" (selva) y la forestación. Es así que encontramos que

"los vecinos hablaban de la florestación como una selva en la cual no hay lugar para nada de la vida humana que actualmente conocen: ni el ganado, ni ellos mismos y sus actividades cotidianas, ni la vista del horizonte, que se ve limitada por la barrera oscura de los árboles plantados. Lo que puede parecer una añoranza romántica (la preocupación paisajística de no poder ver el horizonte) es en realidad una profunda preocupación social: es no poder ver la casa del vecino a lo lejos, una señal de auxilio o un saludo, la llegada de un vehículo o un paisano a caballo por el campo. Es la preocupación por el aislamiento que la despoblación y el nuevo destino productivo -las plantaciones de eucaliptos-generarían en la zona". (Santos, C. 2020, p. 101)

El equipo fue muy cauteloso para no caer en la frecuente asunción de que una crítica tan profunda al modelo de monocultivo forestal basada en un lenguaje de valoración antagónico al dominante fuera equivalente a un inminente conflicto ambiental. Como sostienen Gavazzo et al., no se puede asumir que la conflictividad ambiental sea un tema central aun cuando los actores locales expresen sus opiniones formuladas en términos ambientalizados. En su actividad con trabajadores de una planta de reciclaje de residuos encontraron que:

"en el día a día (...) 'lo ambiental' no era un tema, nadie comentaba ni se preocupaba por eso. Solo si indagábamos podía haber menciones sobre algunas reacciones cutáneas al contacto con la basura o acerca del agua que consumen. Es decir, existe como problemática pero no es prioritaria”. (Gavazzo et al., 2018)

12) Joan Martínez Alier propone analizar las discrepancias de valoración que suceden en los conflictos ambientales (o ecológicos redistributivos, como prefiere denominarlos el autor). En ese sentido, propone que "en algunos conflictos ambientales puede suceder que el conflicto se exprese en una discrepancia de valoración dentro de un único sistema de valoración (habitualmente el monetario) (...) [mientras que en] otros conflictos (...) se manifiesta un pluralismo de valores, existe una disputa sobre el propio sistema de valoración que debe ser empleado. Así sucede cuando se comparan en términos no conmensurables los siguientes valores: la pérdida de biodiversidad, la pérdida de patrimonio cultural, el daño al sustento humano, la pérdida de autonomía o del derecho de autodeterminación local, las violaciones de otros derechos humanos, las ganancias económicas de un proyecto minero, una represa, una camaronera. Hay también un choque de lenguajes de valoración cuando la sacralidad del territorio, la justicia ambiental, los derechos territoriales indígenas o la seguridad ambiental son desplegados contra la valoración monetaria de los riesgos y cargas ambientales" (Martínez-Alier, 2006, p.10). 
Sobre todos los eventuales conflictos (plantaciones forestales, instalación de un parque eólico, prácticas de caza y la propia implementación del área protegida) el equipo desarrolló diferentes iniciativas basadas en el diálogo y la escucha para establecer procesos de construcción de la demanda con los actores locales que colaboran en la identificación de beneficios, perjuicios y alternativas (Gutiérrez et al., 2017).

\section{Reflexiones finales}

El diálogo de saberes expuesto en este trabajo no ha seguido una dirección prefijada, sino que la noción de "ambiente" se considera abierta, objeto de construcción continua. Si bien el monitoreo participativo con cámaras trampa vehiculiza la distinción entre animales silvestres y domésticos y propone tácitamente una cierta idea y valoración de naturaleza, su forma horizontal de diálogo habilita la expresión de otras categorizaciones y percepciones sobre los animales, propias de los habitantes del lugar y no siempre coincidentes con el ideario conservacionista clásico.

La relación entre ambiente y conservación es entendida por el equipo como eminentemente política. Muestra de ello es la pregunta ¿qué queremos conservar?, que ha estado presente en las distintas ediciones del EFI y ha guiado la trayectoria del equipo. Lejos de querer responder a esta cuestión enumerando una serie de "objetos focales" desde la perspectiva de la biología de la conservación, esto pretende abrir las posibilidades cognitivas y narrativas de construcción de problemas socioambientales (Merlinsky, 2018). En lugar de afirmar la jerarquía del conocimiento académico y caer en la "arrogancia del experto" descrita por Fals Borda (1981), se busca generar condiciones para la "coteorización" (Fernández Álvarez y Carenzo, 2012) y la construcción de una "red contraexperta" (Merlinsky, 2018). El conocimiento mutuo entre habitantes y universitarios así como el conocimiento del territorio y el seguimiento de los procesos que lo atraviesan son claves para que este diálogo tenga sentido para todas las partes involucradas.

En la extensión del paradigma de la integralidad, la información ambiental fluye inicialmente desde los universitarios hacia los pobladores. Luego de generar confianza y modestos proyectos comunes (i.e. monitoreo de fauna), los pobladores locales informan y enseñan a los universitarios. Siguiendo el proceso entre el equipo universitario y Paso Centurión, se podría sugerir que es necesario crear mojones (como las actividades en el medio) donde se expliciten las interpretaciones conjuntas acerca de las evidencias y la información ambiental.

Es preciso dejar abierta la noción de "lo ambiental", pues si bien existe ya medio siglo de discusiones que intenta delimitar un campo académico y político asociado a un lenguaje para representar la realidad, la extensión integral debe ser capaz de mantener en latencia las definiciones científicas y originadas en las universidades para escuchar, entender y negociar con los interlocutores una definición colectiva de la coevolución entre sociedad humana y naturaleza externa. Por ejemplo, en Paso Centurión lo ambiental incluye entidades de carácter mágico que pueblan el monte y conviven con la fauna silvestre. También incluye los procesos de migración y despoblamiento. Dicho esto, la ambientalización del territorio puede jugar en contra de la necesaria apertura epistémica de lo ambiental. 
El equipo universitario en este proceso nunca tuvo como meta crear "sujetos ambientalistas" —individuales o colectivos-. Tampoco hallar "conflictos socioambientales" que no fueron percibidos como tales por los interlocutores. De la biología conservacionista a la justicia ambiental, la forma de comprender la intervención de los EFI se vuelve más politizada, quizá más evidente en el equipo universitario que en los pobladores locales. No obstante, en estos momentos, cuando se está discutiendo una reducción de área protegida a instancias de grupos de poder político y económico, la politización de la cuestión ambiental se vuelve explícita y visible e implica a la población local en una lucha por la conservación: la conservación de sus formas de vida.

\section{Referencias bibliográficas}

Barrero, G.; Cardozo, D.; González, M. N.; Grabino, V.; Viñar, M. y Santos, C. (2015). Los proyectos de extensión universitaria. Cuaderno de Extensión 4. Formulación de Proyectos de extensión universitaria. SCEAM, UdelaR, 13-63.

Bergós, L.; Chouhy, M.; Garay, A.; Gaucher, L.; Grattarola, F.; Perazza, G.; Santos, C. y Taks, J. (2019). Participación social, conocimiento experto y conflictos ambientales sobre uso del suelo, energía y biodiversidad en Paso Centurión (Cerro Largo, Uruguay). UdelaR, CSIC, Programa I+D, Montevideo. Informe final (manuscrito). Bergós, L.; Grattarola, F.; Barreneche, J. M.; Hernández, D. y González, S. (2018). Fogones de Fauna: An experience of participatory monitoring of wildlife in rural Uruguay. Society \& Animals, 26(2), 171-185.

Chouhy, M.; Santos, C.; Gaucher, L.; Grattarola, F.; Taks, J.; Bergós, L.; Garay, A. y Perazza, G. (2017). En las fronteras de los saberes: las búsquedas de un Espacio de Formación Integral sobre sociedad-naturaleza. Integralidad Sobre Ruedas, 4(1), 62-77.

Fals Borda, O. (1981). La ciencia y el pueblo. En Vío Grossi, F.; Gianotten, V. y De Wit, T. (Eds.). Investigación participativa y praxis rural: nuevos conceptos en educación y desarrollo comunal (pp. 19-47). Mosca Azul Editores. Fals Borda, O. (2014). Orígenes universales y retos actuales de la IAP (Investigación Acción Participativa). En Herrera Farfán, N. A. y Lopez Guzmán, L. (Comps.). Ciencia, compromiso y cambio social. Textos de Orlando Fals Borda (pp. 265-282). Lanzas y Letras-El Colectivo-Extensión Libros.

Fernández Álvarez, M. I. y Carenzo S. (2012). "Ellos son los compañeros del Conicet”: el vínculo con organizaciones sociales como desafío etnográfico. Publicar en Antropología y Ciencias Sociales, 12, 9-33.

Ferrero, B. G. (2013). La conservación de la naturaleza como arena de acción política. Dos conflictos en la provincia de Misiones. Publicar en Antropología y Ciencias Sociales, 15, 33-54.

Feyerabend, P. (1998). La ciencia en una sociedad libre. Siglo XXI Editores. 1982.

Gautreau, P. (2014). Forestación, territorio y ambiente. 25 años de silvicultura transnacional en Uruguay, Brasil y Argentina. Trilce.

Gavazzo, N.; Espina, R.; Arango, C.; González, S. y Canuto, D. (2018). El diálogo de saberes como estrategia metodológica para la articulación de investigación y extensión. La experiencia del Programa Fals Borda del IDAES. Papeles de trabajo. Revista Electrónica del IDAES, 12(1), 55-72.

Ghiso, A. (1999). Acercamientos: el taller en procesos de investigación interactivo. Estudios sobre las Culturas Contemporáneas. Universidad de Colima, Colima, México, 5(9), 141-153.

Grattarola, F.; Gutiérrez, L.; Garay, A.; Gaucher, L.; Gonzáles Barrios, P.; Perazza, G.; Bergós, L.; Santos, C.; Contreras Sunhary, S.; Chouhy, M. y Cosse, M. (2014). Registro de mamíferos por medio de métodos no invasivos en la reserva natural de Paso Centurión. En Libro de resúmenes del I Congreso Latinoamericano de Genética para la Conservación.

Revista +E 11 (14) | 2021 | pp. 1-14 | Santos, C.; Chouhy, M.; Bergós, L. y Garay, A. (2021) | Ambiente y extensión en Paso... 
Grattarola, F.; Hernández, D.; Duarte, A.; Gaucher, L.; Perazza, G.; González, S.; Bergós, L.; Chouhy, M.; Garay, A.; Carabio, M. y Rodríguez-Tricot, L. (2016). Primer registro de yaguarundí (Puma yagouaroundi) (MAMMALIA: CARNIVORA: FELIDAE) en Uruguay, con comentarios sobre monitoreo participativo. Boletín de la Sociedad Zoológica del Uruguay (2da. época), 25(1), 85-91.

Grattarola, F. y Rodríguez-Tricot, L. (2020). Mammals of Paso Centurión, an area with relicts of Atlantic Forest in Uruguay. Neotropical Biology and Conservation, 15(3), 267-283.

Gutiérrez, M.; Yanniello, F.; Elisio, S. y Andrionolo, D. (2017). La extensión universitaria como herramienta de intervención en los conflictos socioambientales. +E Revista de Extensión Universitaria, 7(7), 204-213.

Ingold, T. (2012). Ambientes para la vida. Conversaciones sobre humanidad, conocimiento y antropología. Trilce-FHUCE-Extensión universitaria.

La voz de Melo (27 de febrero de 2021). El futuro del área protegida de Centurión será analizado entre vecinos de la zona. http://lavozdemelo.com

Leite Lopes, J. S. (2006). Sobre Processos de "ambientalização" dos conflitos e sobre dilemas da participação. Horizontes Antropológicos, 25, 31-64.

Martínez-Alier, J. (2006). Los conflictos ecológico-distributivos y los indicadores de sustentabilidad. POLIS, Revista de la Universidad Bolivariana, 5(13), 0.

Merlinsky, G. (2018). Justicia ambiental y políticas de reconocimiento en Buenos Aires. Perfiles latinoamericanos, 26(51), 241-263. FLACSO.

Pombo, O. (2013). Epistemología de la interdisciplinariedad. La construcción de un nuevo modelo de comprensión. Interdisciplina, 1, 21-49. Universidad Nacional Autónoma de México.

Quintana, M.; Capurro, P. y González Fajardo, P. (2019). Hacia un pensar colectivo. Una experiencia de extensión universitaria en las ruralidades uruguayas. +E: Revista de Extensión Universitaria, 9(10), 108-123. 10.14409/extension.v9i10

Retamozo, M. (2015). Las demandas sociales y el estudio de los movimientos sociales. Cuaderno de Extensión 4. Formulación de Proyectos de extensión universitaria. SCEAM. Universidad de la República. Montevideo, 101-132.

Santos, B. de S. (2009). Más allá del pensamiento abismal: de las líneas globales a una ecología de saberes. Una epistemología del sur. La reinvención del conocimiento y la emancipación social. Siglo XXI Editores. CLACSO.

Santos, C. (2020). Naturaleza y hegemonía progresista. Los conflictos ambientales en los gobiernos del Frente Amplio en Uruguay. Gorla-Pomaire.

Santos, C.; Bergós, L.; Chouhy, M.; Garay, A.; Gaucher, L.; Grattarola, F.; Perazza, G. y Taks, J. (2020). Un ambiente para la integralidad. Lecciones aprendidas a partir de una experiencia interdisciplinaria. Ciencias sociales y extensión universitaria: aportes para el debate, Vol. 2. Facultad de Ciencias Sociales, UdelaR.

Santos, C.; Prol, L. y Bergós, L. (2019). Co-producción de conocimientos e injusticias socio-ambientales. Reflexiones a partir de dos experiencias en Argentina y Uruguay. Fronteras, (12), 115-128.

Taks, J. (2013). Los desafíos de la antropología para la comprensión de los conflictos socio-ambientales en Sudamérica. En Machado, C.; Dos Santos, C.; Ferreira Araújo, C. y Dos Passos, W. (Eds.). Conflitos Urbanos e Ambientais: debates, lutas e desafios. FURG, 21-40.

Tommasino, H.; Cano, A.; Castro, D.; Santos, C. y Stevenazzi, F. (2010). De la extensión a las prácticas integrales. La Extensión en la Renovación de la Enseñanza: Espacios de Formación Integral. Hacia la Reforma Universitaria, 10, 25-31.

UdelaR (2019). Convocatoria para el Apoyo a Actividades en el Medio 2019 - Cierre extraordinario. Bases. http://www.extension.udelar.edu.uy/wp-content/uploads/2019/05/Punto-8_Convocatoria-y-bases-Apoyo-Actividades-en-el-Medio-2019_Cierre-EXTRAORDINARIO.pdf 